\title{
Factores asociados al peso del aditamento de universitarios
}

\author{
Factors associated with the weight of the backpack of university students \\ Rocío Pizarro-Andrade \\ Melina Cruzado-Melendez ${ }^{1}$
}

\begin{abstract}
Resumen
Objetivo: determinar la asociación entre el peso del aditamento y sus factores asociados en universitarios. Materiales y métodos: estudio correlacional de corte transversal. El tamaño de muestra fue 226 estudiantes. Se consideró peso inadecuado cuando es mayor (5\%) del peso corporal. Se emplearon las pruebas estadísticas de Kruskal Wallis y Tstudent. Resultados: el 79,8\% de los encuestados usaba mochila y el 50\% cargaba un peso inadecuado en su aditamento. El $60.2 \%$ de los universitarios presentó dolor, de ellos $40.2 \%$ en la zona cervical-lumbar. El $56.6 \%$ que manifestó dolor reportó carga inadecuada del aditamento $(\mathrm{p}=0.014)$. Mientras que el $66.2 \%$ de los encuestados manifestó intensidad moderada de dolor llevando un peso inadecuado del aditamento ( $\mathrm{p}=0.027)$. El $70 \%$ de los estudiantes que manifestó dolor en la zona cervical llevaba un peso inadecuado $(\mathrm{p}=0.049)$. Por el contrario, se encontró que el $40 \%$ de $\operatorname{los}$ participantes que tenía dolor en las zonas del cervical y lumbar llevaba un peso adecuado $(\mathrm{p}=0.049)$. Por último, el $55 \%$ de integrantes que usaba mochila llevaba un peso inadecuado $(\mathrm{p}=0.002)$. Conclusión: el peso inadecuado del aditamento está relacionado con la presencia de dolor y con una intensidad de dolor moderada. También se asoció las zonas de dolor (cervical) y el peso inadecuado del bolso. Asimismo, se halló relación entre las zonas de dolor (cervical-lumbar) y el peso adecuado. Se encontró relación entre el tipo de bolso y peso del mismo.
\end{abstract}

Palabras clave: Dolor musculoesquelético; Postura; Estudiantes; Peso corporal (Fuente: DeCS).

\begin{abstract}
Objective: to determine the association between the weight of the book bag and the associated factors in university students. Materials and methods: cross-sectional correlational study. The sample size was 226 students. Inadequate weight was considered when it is greater $(5 \%)$ of body weight. The statistical tests of Kruskal Wallis and T-student were used. Results: $79.8 \%$ of the respondents wore a backpack and $50 \%$ carried an inadequate weight in their book bag. The $60.2 \%$ of the university students presented pain, of them $40.2 \%$ in the cervical-lumbar area. $56.6 \%$ of university students who reported pain reported inadequate loading of the abutment $(\mathrm{p}=0.014)$. While $66.2 \%$ of the respondents showed moderate intensity of pain carrying an inadequate weight of the attachment $(\mathrm{p}=0.027) .70 \%$ of the students who showed pain in the cervical area were carrying an inadequate weight $(\mathrm{p}=0.049)$. On the contrary, it was found that $40 \%$ of the participants who had pain in the cervical and lumbar areas carried an adequate weight $(\mathrm{p}=0.049)$. Finally, 55\% of members who used a backpack carried an inadequate weight $(\mathrm{p}=0.002)$. Conclusions: the inadequate weight of the book bag is related to the presence of pain and moderate intensity of pain. The areas of pain (cervical) and the inadequate weight of the bag were also associated. Likewise, a relationship was found between the areas of pain (cervical-lumbar) and adequate weight. A relationship was found between the type of bag and its weight.
\end{abstract}

Key words: Musculoskeletal pain; Position; Students; Body weight (Source: DeCS).

Para citar:

Pizarro R, Cruzado M. Factores asociados al peso del aditamento de universitarios. CASUS. 2018;3(2):103-109.

\footnotetext{
${ }^{1}$ Facultad de Ciencias de la Salud, Universidad Católica Sedes Sapientiae. Licenciada en Tecnología Médica en Terapia Física y Rehabilitación. Correo electrónico: rpizarro@ucss.edu.pe
}

Fecha de recepción: 04-06-18

Fecha de envío a pares: 05-06-18

Fecha de aprobación por pares: 27-07-18

Fecha de aceptación: 08-08-18 


\section{INTRODUCCIÓN}

En la etapa universitaria se utilizan como medio de transporte diversos aditamentos para trasladar materiales de estudio, trabajo, entre otros (1). El uso del bolso permite el transporte de una considerable cantidad de materiales. Estudios manifiestan que el $70 \%$ de las adolescentes y el $50 \%$ de los adolescentes varones presentan dolor provocado por el peso del bolso (2-6). Por ello, la Organización Mundial de la Salud (OMS) en los últimos 20 años apoya la investigación de los factores y comportamientos que influyen en la salud en los adolescentes. Además, alerta que entre las principales causas de discapacidad se encuentra el dolor de espalda y cuello.

Por otro lado, el estudio Global Burden of Diseases, Injuries and Risk Factors (GBD) realizó evaluaciones de prevalencia, incidencia y años vividos con discapacidad. Posteriormente elaboró una lista de enfermedades y lesiones de todos los países desde el año 1990 hasta el 2016 incluyendo al dolor lumbar y cervical entre las cinco principales causas de discapacidad músculoesquelética (7). Asimismo, una revisión sistemática que incluía estudios europeos, de Oceanía, Asia, África y América del Norte reportó la presencia del dolor lumbar asociada a diversos factores, entre ellos el peso del bolso (8). De la misma manera, se ha reportado la influencia del tipo y la forma de uso del aditamento en la presencia de dolor (9). No obstante la coincidencia en este aspecto, existe divergencia en relación a las recomendaciones del peso del aditamento. Las cuales varían entre el $10 \%$ y el $25 \%$ del peso corporal $(10,11)$. Sin embargo, se han evidenciado cambios en los ángulos de la columna vertebral (12) con un peso del aditamento inferior al $10 \%$ del peso corporal (10).

La población universitaria es vulnerable dado que en la mayoría de casos presenta alteraciones posturales previas que son adquiridas en la etapa escolar o infancia (13). Además, los universitarios cumplen con actividades extracurriculares como realizar deportes, trabajar, entre otros. Estas actividades aumentan el tiempo de carga y peso del bolso produciendo así compensaciones. La combinación de estos factores: las alteraciones posturales previas, el tiempo de carga y peso del bolso pueden desencadenar dolor (8). Se debe considerar, además, que el dolor produce un impacto negativo en los determinantes de la salud como en el incremento de los costos en el sistema de salud, limitaciones en las actividades funcionales, cambios en el aspecto psicológico y alteraciones en la calidad de vida (14-16).

Por las razones anteriormente mencionadas es relevante continuar indagando en las diversas causas de la presencia de dolor en los universitarios, entre ellas el peso inadecuado del bolso. Diversos estudios en universitarios $(10,11)$ indagan sobre la relación entre el llevar pesos de $10 \%$ al $25 \%$ del peso corporal y el dolor musculoesquelético. Teniendo en cuenta que se pueden evidenciar cambios estructurales en la curvatura espinal con cargas superiores al 5\% del peso corporal, aún queda por profundizar en los factores asociados a la presencia del dolor con pesos inferiores a lo recomendado. Por ello, la finalidad del estudio fue determinar los factores asociados al peso del aditamento con el 5\% del peso corporal.

\section{MATERIALES Y MÉTODOS}

Estudio correlacional de corte transversal en universitarios de Lima Norte y de Tarma. El tamaño de muestra fue 226 estudiantes de 16 a 47 años de edad, se calculó con una precisión de 3\%, una proporción de $5 \%$ y una proporción esperada de pérdidas de $10 \%$. El muestreo fue no probabilístico por conveniencia. Se incluyeron universitarios del $1^{\circ}$ al $10^{\circ}$ ciclo, de ambos sexos y se excluyeron aquellos que no usaban aditamento o que presentan alguna disfunción del movimiento corporal.

La variable principal fue peso de aditamento definida como la cantidad de masa que se transporta en una bolsa y/o mochila. De acuerdo a estudios previos, la carga máxima que debería tener la mochila para que sea segura no debe superar el 15\%-25\% del peso corporal, sin embargo para esta investigación se trabajó con el $5 \%$ del peso corporal (10). Se utilizó como 
instrumento una balanza mecánica, donde el rango de peso máximo fue $15 \mathrm{~kg}$. Variable cualitativa dicotómica: peso adecuado del aditamento (menor o igual a $5 \%$ del peso corporal) y peso inadecuado de aditamento (mayor al 5\% del peso corporal).

Igualmente se evaluó la presencia de dolor. Variable cualitativa dicotómica (sí y no). Asimismo, se evaluó la intensidad del dolor. Se usó como instrumento la Escala Análoga Visual del Dolor (EVA). Variable cualitativa policotómica: dolor leve (1-3), dolor moderado (46) y dolor intenso $(7-10)(17,18)$. También, se evaluó la zona de dolor, siendo una variable cualitativa policotómica: cervical, hombro, dorsal, lumbar, cervical-lumbar y otras zonas. Por otro lado, se consideró el tipo de aditamento, siendo una variable cualitativa policotómica: mochila, maletín, cartera y morral, y también la forma de uso del aditamento siendo una variable cualitativa policotómica: en un hombro, dos hombros, cruzado y en la mano. Por último, variable tiempo de uso, variable cualitativa policotómica; 5min$15 \mathrm{~min}, 15 \mathrm{~min}-30 \mathrm{~min}, 30 \mathrm{~min}-60 \mathrm{~min}$ y más de 60 min.

Por otro lado, se consideraron variables sociodemográficas tales como edad, variable cuantitativa (de 16 a 47 años), sexo, variable cualitativa dicotómica (masculino, femenino), carrera, variable cualitativa policotómica (Ciencias Comerciales y Económicas, Ciencias de la Salud, Ingeniería e Ingeniería Agraria) y ciclo, variable cualitativa policotómica (I-III, IV-VI, VII$X)$. Toda la información fue recolectada en una "Ficha de recolección de datos".

Los datos obtenidos fueron digitados en el software Microsoft Excel 2010 (Microsoft, US), para posteriormente ser exportados a una base datos al programa STATA versión 14. En el análisis descriptivo de las variables categóricas se emplearon frecuencias y porcentajes. Mientras que para las variables numéricas medidas de tendencia central. Las asociaciones fueron evaluadas con las pruebas estadísticas de Kruskal Wallis y T-student. La información se expresó mediante las tablas de contingencia con una significancia menor o igual a

0.05. Asimismo, para obtener el consentimiento a la participación se brindó información relevante sobre las características del estudio y posteriormente, sobre algunos resultados preliminares del mismo. Con la finalidad de proteger la identidad de los encuestados todos los datos personales recolectados fueron codificados.

\section{RESULTADOS}

En la tabla 1 se observa que la media de edad de los universitarios fue de 21 años y el promedio aproximado del peso del aditamento fue de $3 \mathrm{~kg}$. El $57.59 \%$ de los encuestados era de sexo femenino, el $53.54 \%$ proveniente de la carrera de ingeniería e ingeniería agraria, el $79.82 \%$ usaba mochila, el 50\% cargaba un peso inadecuado en su aditamento, mientras que el $60.18 \%$ presentó dolor y el 59.13\% reportó una intensidad moderada de dolor. Por último, el $40.18 \%$ de los universitarios presentó dolor en la zona cervical-lumbar de la columna vertebral.

Tabla 1. Características descriptivas

\begin{tabular}{|c|c|c|c|}
\hline \multirow{2}{*}{ Edad* } & & $\mathbf{n}$ & $\%$ \\
\hline & & 21 & 4.3 \\
\hline Sexo & Masculino & 95 & 42.4 \\
\hline & Femenino & 129 & 57.6 \\
\hline \multicolumn{4}{|c|}{ Carrera } \\
\hline & Ciencias & & \\
\hline & $\begin{array}{l}\text { Comerciales } \\
\text { y Económicas }\end{array}$ & 32 & 14.1 \\
\hline & $\begin{array}{l}\text { Ciencias de la } \\
\text { Salud }\end{array}$ & 73 & 32.3 \\
\hline & Ingeniería e & & \\
\hline & $\begin{array}{l}\text { Ingeniería } \\
\text { Agraria }\end{array}$ & 121 & 53.5 \\
\hline \multicolumn{4}{|l|}{ Ciclo } \\
\hline & I-III & 69 & 31.5 \\
\hline & IV-VI & 114 & 52.0 \\
\hline & VII-X & 36 & 16.4 \\
\hline Peso c & rporal* & 60.52 & 10.6 \\
\hline Peso d & aditamento* & 3.19 & 1.2 \\
\hline \multicolumn{4}{|c|}{ Peso de aditamento } \\
\hline & $\begin{array}{l}\text { Peso } \\
\text { inadecuado }\end{array}$ & 113 & 50.0 \\
\hline & $\begin{array}{l}\text { Peso } \\
\text { adecuado }\end{array}$ & 113 & 50.0 \\
\hline \multicolumn{4}{|c|}{ Tipo de aditamento } \\
\hline & Mochila & 178 & 79.8 \\
\hline & Maletín & 8 & 3.6 \\
\hline & Cartera & 19 & 8.5 \\
\hline & Morral & 18 & 8.0 \\
\hline
\end{tabular}


Tabla 1. continúa

\begin{tabular}{|c|c|c|}
\hline & n & $\%$ \\
\hline \multicolumn{3}{|l|}{ Forma de uso } \\
\hline En un hombro & 95 & 42.2 \\
\hline En dos hombros & 120 & 53.3 \\
\hline Cruzado & 4 & 1.7 \\
\hline Mano & 6 & 2.7 \\
\hline \multicolumn{3}{|l|}{ Tiempo de uso } \\
\hline $5 \min -15 \mathrm{~min}$ & 43 & 19.8 \\
\hline $15 \mathrm{~min}-30 \mathrm{~min}$ & 81 & 37.3 \\
\hline 30min-60min & 74 & 34.1 \\
\hline Más de $60 \mathrm{~min}$ & 19 & 8.7 \\
\hline \multicolumn{3}{|l|}{ Dolor } \\
\hline Sí & 136 & 60.2 \\
\hline No & 90 & 39.8 \\
\hline \multicolumn{3}{|l|}{ Intensidad de dolor } \\
\hline Dolor leve & 26 & 22.6 \\
\hline Dolor moderado & 68 & 59.1 \\
\hline Dolor severo & 21 & 18.2 \\
\hline \multicolumn{3}{|l|}{ Zona de dolor } \\
\hline Otras zonas & 39 & 17.4 \\
\hline Cervical & 10 & 4.4 \\
\hline Hombro & 49 & 21.8 \\
\hline Dorsal & 13 & 5.8 \\
\hline Lumbar & 23 & 10.2 \\
\hline Cervical-Lumbar & 90 & 40.18 \\
\hline \multicolumn{3}{|l|}{ Sede } \\
\hline Lima & 100 & 44.2 \\
\hline Tarma & 126 & 55.7 \\
\hline
\end{tabular}

*media y desviación estándar

Se encontró asociación entre el peso del aditamento y la presencia de dolor $(\mathrm{p}=0.014)$. El $56.62 \%$ de los universitarios que manifestó dolor reportó carga inadecuada del aditamento. El $66.18 \%$ de los encuestados que manifestó intensidad de dolor en una escala de moderado llevó peso inadecuado del aditamento $(\mathrm{p}=0.027)$. Igualmente, el $70 \%$ de los estudiantes que manifestó dolor en la zona cervical llevó un peso inadecuado del aditamento $(\mathrm{p}=0.049)$. Por el contrario, se encontró que el $60 \%$ de los participantes que tenía dolor en la zona cervicallumbar llevaba un peso adecuado en el aditamento. Por último, el $55.06 \%$ de integrantes que usaba mochila cargaba un peso inadecuado del aditamento $(\mathrm{p}=0.002)$. Los demás datos se pueden observar en la tabla 2.

\section{DISCUSIÓN}

Los resultados evidenciaron relación entre la presencia de dolor y el peso inadecuado del
Tabla 2. Peso del aditamento y sus factores asociados

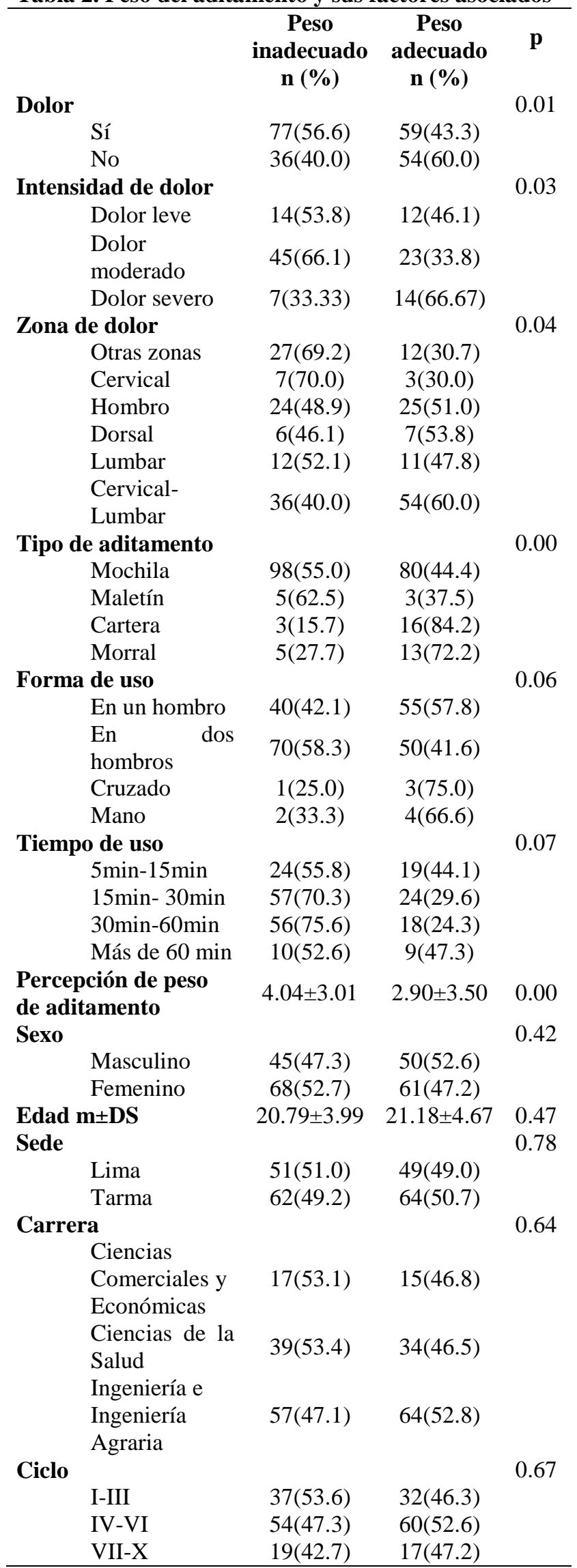


aditamento. Los encuestados que manifestaron intensidad de dolor moderado transportaban mayor peso del aditamento. Igualmente, se observa significancia estadística entre las zonas cervical y lumbar y el peso inadecuado del bolso. Asimismo, se halló relación entre las zonas de cervical-lumbar y el peso adecuado del bolso, al igual que en las zonas de la columna dorsal y hombro. Se encontró relación entre el tipo de bolso y peso del mismo. Los estudiantes que llevaban mochila transportaba mayor peso de lo recomendado.

Se hallaron dos asociaciones estrechamente vinculadas: entre la presencia de dolor y el peso del aditamento, así como entre la intensidad del dolor y el peso del aditamento. Más de la mitad de los participantes que manifestaron dolor, portaron mayor peso de lo recomendado en su aditamento. Así como se reportó asociación entre la intensidad moderada del dolor y el peso inadecuado del bolso. En un estudio (19) se halló que la carga del aditamento contribuye a la presencia del dolor en diversos segmentos corporales de los encuestados. Mientras que por otro lado se informó que a medida que se aumenta la carga del bolso incrementa la intensidad del dolor $(20,21)$. El dolor actúa como un factor protector para evitar daños tisulares (22). Cuando la tensión es intensa se estimulan diversos receptores que producen dolor (23). Un estímulo intenso causará el incremento del dolor. Son numerosos los factores implicados en la presencia e intensidad del dolor, como factores biomecánicos, fisiológicos, emocionales y externos; sin embargo, estos factores no son independientes quedando espacio a interacciones entre estos (24).

Se observó significancia estadística entre la zona del dolor y el peso inadecuado del aditamento. Más de la mitad de los universitarios que expresaron dolor en la zona cervical, llevaban un peso inadecuado en su aditamento, al igual que aquellos que reportaron dolor en la zona lumbar. Estos hallazgos tienen similitud con estudios (25, 26) que describen cambios biomecánicos en la columna cervical y lumbar con respecto al peso inadecuado del bolso. Este cambio va asociado a compensaciones estructurales o musculares que consecuentemente conllevan a dolor en la columna cervical y lumbar (27). Este fenómeno ha sido reportado igualmente en la etapa escolar $(28,26)$.

Del mismo modo, se halló asociación entre la zona de dolor y el peso adecuado del bolso. Se encontraron más participantes que presentaron dolor en las zonas cervical-lumbar y transportaban una carga recomendada en su bolso, al igual que en la zona dorsal y del hombro. En diversos estudios $(9,10)$ se reportó que los cambios estructurales de la columna vertebral y demás segmentos se asocian con transportar pesos menores o mayores a lo recomendado, como respuesta se manifiestan compensaciones que conllevarían al dolor.

Considerando los dos últimos hallazgos se puede inferir que, desde el punto de vista biomecánico, la carga de peso transportada en un aditamento cambia el centro de gravedad corporal, como respuesta se desarrollan una serie de compensaciones que conducen a rigidez en los músculos posturales y tensión en los ligamentos. Estas compensaciones pueden manifestarse con dolor (29).

Por último, se encontró asociación entre el tipo y peso del aditamento. Más universitarios usaban mochila y cargaban un peso inadecuado en el aditamento. En una revisión sistemática se encontró que en gran porcentaje de estudiantes que usaba mochila y además llevaba un peso no recomendado en su aditamento se incrementó el dolor y su intensidad producto de compensaciones estructurales del cuerpo $(9,17)$. El uso de la mochila es tradicional desde la etapa escolar, es de asumir que se da continuidad en la etapa universitaria debido a que proporciona mayores facilidades en el transporte de una considerable cantidad de materiales de estudio a pesar que ello pudiera llevar a posturas compensatorias.

Entre las principales limitaciones del estudio se menciona que por sus particularidades no fue posible establecer el promedio del peso del aditamento durante un período de tiempo, además no se contemplaron variables confusoras como las características de las mochilas. Por último, no se 
realizaron pruebas de diagnóstico para evidenciar zona dolorosa. Cabe mencionar que se trabajó con el 5\% del peso del aditamento, es decir, con un porcentaje inferior a lo descrito en otros estudios (10). Conectado a lo anterior, es uno de los primeros estudios realizados en Perú en población universitaria donde se determinan factores asociados al peso inadecuado del aditamento.

\section{CONCLUSIONES}

En universitarios el uso de aditamento tipo mochila se relaciona con transportar un peso inadecuado del mismo. El peso inadecuado del aditamento se relaciona con la presencia de dolor de intensidad moderada. Asimismo, llevar un peso del aditamento mayor a lo recomendado se relaciona con la presencia de dolor en la zona cervical y lumbar. Sin embargo, llevar un peso adecuado del aditamento también se relaciona con dolor en dos segmentos combinados como la columna cervical-lumbar, dorsal y hombro como respuesta a una serie de compensaciones estructurales. Este hallazgo sugiere que la presencia del dolor en los segmentos mencionados es independiente a la carga del aditamento.

Los resultados expuestos deben complementarse con el análisis de los diversos factores implicados en la producción del dolor, como factores anatómicos, fisiológicos o ambientales. Se recomienda para futuras investigaciones profundizar en la forma de uso del aditamento y la relación entre la forma de las compensaciones posturales en la población. También futuros estudios deben incrementar la dimensión de la población de estudio, determinar el peso promedio del aditamento en un período de tiempo y su relación con diversos factores. Estos aspectos deben investigarse para planificar estrategias preventivas y de rehabilitación adecuadas. Así también, se recomienda a los universitarios limitar el peso del aditamento y así evitar transportar cargas inútiles, organizar y repartir el peso de los materiales en el bolso. Por último, se recomienda a las instituciones educativas brindar información pertinente sobre el uso y peso adecuado del bolso.

\section{REFERENCIAS BIBLIOGRÁFICAS}

1. Rodrigues FB, Magnani RM, Lehnen GC, Souza GS de S e, Andrade AO, Vieira MF. Effects of backpack load and positioning on nonlinear gait features in young adults. Ergonomics. 2018;61(5):720-8.

2. Golriz S, Walker B. Can load carriage system weight, design and placement affect pain and discomfort? A systematic review. J Back Musculoskelet Rehabil. 2011;24(1):1-16.

3. Duggleby T, Kumar S. Epidemiology of juvenile low back pain: A review. Disabil Rehabil. 1997;19(12):505-12.

4. Alshagga MA, Nimer AR, Yan LP, Ibrahim IAA, Al-Ghamdi SS, Radman Al-Dubai SA. Prevalence and factors associated with neck, shoulder and low back pains among medical students in a
Malaysian Medical College. BMC Res Notes. 2013;6:244.

- Camargo Lemosa,D.M, Orozco Vargas L.C , Hernández Sáncheza 8. J. \& Niño Cruza G.I. Dolor de espalda crónico y actividad física en estudiantes universitarios de áreas de la salud. Rev Soc Esp Dolor. 2009;16(8):429-436.

6. Casas Sánchez A.S \& Patiño 9 Segura M.S. Prevalencia y factores asociados con el dolor de espalda y cuello en estudiantes universitarios. Salud UIS. 2012; 44 (2): 45-55.

7. GBD 2016 Disease and Injury Incidence and
Collaborators.Global, and national incidence, prevalence, and years lived with disability for 328 diseases and injuries for 195 countries, 19902016: a systematic analysis for the Global Burden of Disease Study
2016. Global Health Metrics; 2016 Volume 390, No. 10100, p12111259 ,

Robalo L, Cruz E \& Nunes C. Epidemiology of Non-Specific Back Pain in Children and Adolescents: a Systematic Review of Observational Studies.J Nov Physiother. 2015;5:266.

Kimberly D. Dahl He Wanga , Jennifer K. Popp D, Clark Dickin. Load distribution and postural changes in young adults when wearing a traditional backpack versus the BackTpack. Gait \& Posture. 2016; 45: 90-96.

10. Simon S. W. Li \& Daniel H. K. Chow. Multi-objective analysis for assessing simultaneous changes in regional spinal curvatures under backpack carriage in young adults. Ergonomics.J Nov Physiother. 2015; 5:266. 
11. Devroey K.C, Jonkers I, An de 17. Sabine J. G. M, Aletta M, Becker, Lenaerts G \& Spaepen A. Evaluation of the effect of backpack load and position during standing and walking using biomechanical, physiological and subjective

measures.Ergonomics.2007;50:5,7 28-742.

12. Min-hee K and Won-gyu Y. Effect of the Spacing of Backpack Shoulder Straps on Cervical Muscle Activity, Acromion and Scapular Position, and Upper Trapezius Pain. J Phys Ther Sci. 2013; 25(6): 685-686.

13. Brackley HM, Stevenson JM, Selinger JC. Effect of backpack load placement on posture and spinal curvature in prepubescent children. Work Read Mass. 2009;32(3):351-60.

14. Nketia-Kyere M, Aryeetey GC, Nonvignon J, Aikins M. Exploring barriers to accessing physiotherapy services for stroke patients at Tema general hospital, Ghana. Archives of Physiotherapy. 2017;7:8.

15. Passalent LA, Landry MD, Cott CA. Exploring Wait List Prioritization and Management Strategies for Publicly Funded Ambulatory Rehabilitation Services in Ontario, Canada: Further Evidence of Barriers to Access for People with Chronic Disease. Healthc Policy. 2010;5(4):139-56.

16. Alföldi P, Wiklund T, Gerdle B. Comorbid insomnia in patients with chronic pain: a study based on the Swedish quality registry for pain rehabilitation (SQRP). Disabil Rehabil. 2014;36(20):1661-9.
Monique ,Tibboel D, Catherine A. J. The Use of the Behavioral Pain Scale to Assess Pain in Conscious Sedated Patients. Critical Care and Trauma. 2010 ; Vol. 110, No. 1.

18. Köppen PJ, Dorner TE, Stein KV, Simon J, Crevenna R. Health literacy, pain intensity and pain perception in patients with chronic pain. Wiener Klinische Wochenschrift. $\quad 2018 ; 130(1): 23$ 30 .

19. Adeyemi AJ, Rohani JM, Abdul Rani MR. Backpack-back pain complexity and the need for multifactorial safe weight recommendation. Appl Ergon. 2017;58:573-582.

20. Deepak S, Ajeesh P.S, Jose, Jerrish A. Debnath, Sukrit. Manjula, M. Backpack injuries in Indian school children: risk factors and clinical presentations. Work. 2012 vol. 41, no. Supplement 1, pp. 929-932.

21. Samira Golriz and Bruce Walker. Can load carriage system weight, design and placement affect pain and discomfort? Asystematic review. Journal of Back and Musculoskeletal Rehabilitation. 2011; 1(16).

22. Moreno C, Prada D.M. Fisiopatología del dolor clínico. ACN.2004; cap 2.

23. Rico M.A. Pathophysiology of chronic musculoskeletal pain. Medwave. 2008;8(8).

24. Overton M, Du Plessis H, Sole G. Electromyography of neck and shoulder muscles in instrumental musicians with musculoskeletal pain compared to asymptomatic controls: A systematic review and meta-analysis. Musculoskelet Sci Pract. 2018;36:32-42.

25. Cheung C.H, Shum S.T, Tang S.F , Yau P.C. and Thomas T.W. Chiu. The correlation between craniovertebral angle, backpack weights, and disability due to neck pain in adolescents. Journal of Back and Musculoskeletal Rehabilitation. 2010;129-136.

26. Christa Devroey, Ilse Jonkers, An de Becker, Gerlinde Lenaerts \& Arthur Spaepen. Evaluation of the effect of backpack load and position during standing and walking using biomechanical, physiological and subjective measures, Ergonomics. 2017; 50:5, 728-742.

27. Patrick D, Wettenschwiler, Lorenzetti S, Stephen J, Ferguson, Stämpfli R, Ameet K. Aiyangar, René M. Rossi \& Simon Annaheim. Loading of the lumbar spine during backpack carriage,Computer Methods in Biomechanics and Biomedical Engineering. 2016; 20:5, 558-565.

28. Rodríguez-Oviedo P, RuanoRavina A, Pérez-Ríos M.School children's backpacks, back pain and back pathologies Archives of Disease in Childhood 2012;97:730-732.

29. Rosangela B. Macedo, Manuel J. Coelho-e-Silva, Nuno F. Sousa, João Valente-dos-Santos, Aristides M. Machado-Rodrigues, Sean P. Cumming, Alessandra V. Lima, Rui S. Gonçalves, Raul A. Martins. Quality of life, school backpack weight, and nonspecific low back pain in children and adolescents. Jornal de Pediatria. 2015; 263-269. 